\title{
Safety and Angiographic Efficacy of Intra-Arterial Fibrinolytics as Adjunct to Mechanical Thrombectomy: Results from the INFINITY Registry
}

\author{
Johannes Kaesmacher, ${ }^{\mathrm{a}, \mathrm{b}, *}$ Nuran Abdullayev, ${ }^{\mathrm{c}, *}$ Basel Maamari, ${ }^{\mathrm{d}}$ Tomas Dobrocky, ${ }^{\mathrm{a}}$ Jan Vynckier, ${ }^{\mathrm{d}}$ \\ Eike I. Piechowiak, ${ }^{\text {a }}$ Raoul Pop, ${ }^{\mathrm{e}}$ Daniel Behme, ${ }^{\mathrm{f}}$ Peter B. Sporns, ${ }^{\text {gh }}{ }^{\mathrm{h}}$ Hanna Styczen, ${ }^{\mathrm{i}}$ Pekka Virtanen, ${ }^{\mathrm{j}}$ \\ Lukas Meyer, ${ }^{\mathrm{h}}$ Thomas R. Meinel, ${ }^{\mathrm{d}}$ Daniel Cantré, ${ }^{\mathrm{k}}$ Christoph Kabbasch, ${ }^{\mathrm{c}}$ Volker Maus, ${ }^{\mathrm{l}}$ Johanna Pekkola, ${ }^{\mathrm{j}}$ \\ Sebastian Fischer, ${ }^{\mathrm{l}}$ Anca Hasiu, ${ }^{\mathrm{e}}$ Alexander Schwarz, ${ }^{\mathrm{f}}$ Moritz Wildgruber, ${ }^{\mathrm{m}, \mathrm{n}}$ David J. Seiffge, ${ }^{\mathrm{d}}$ \\ Sönke Langner, ${ }^{\mathrm{k}}$ Nicolas Martinez-Majander, ${ }^{\mathrm{o}}$ Alexander Radbruch, ${ }^{\mathrm{i}, \mathrm{p}}$ Marc Schlamann, ${ }^{\mathrm{c}}$ Dan Mihoc, \\ Rémy Beaujeux, ${ }^{\mathrm{e}}$ Daniel Strbian, ${ }^{\mathrm{o}}$ Jens Fiehler, ${ }^{\mathrm{h}}$ Pasquale Mordasini, ${ }^{\mathrm{a}}$ Jan Gralla, ${ }^{\mathrm{a}}$ Urs Fischer ${ }^{\mathrm{d}}$ \\ aniversity Institute of Diagnostic and Interventional Neuroradiology, University Hospital Bern, Inselspital, University of Bern, Bern, Switzerland \\ 'University Institute of Diagnostic and Interventional and Pediatric Radiology, University Hospital Bern, Inselspital, University of Bern, Bern, \\ Switzerland \\ Institute for Diagnostic and Interventional Radiology, Faculty of Medicine and University Hospital Cologne, Cologne, Germany \\ ${ }^{d}$ Department of Neurology, University Hospital Bern, Inselspital, University of Bern, Bern, Switzerland \\ eDepartment of Interventional Neuroradiology, University Hospital Strasbourg, Strasbourg, France \\ fDepartment of Neuroradiology, University Hospital Göttingen, Göttingen, Germany \\ ${ }^{9}$ Department of Neuroradiology, University Hospital Basel, Basel, Switzerland \\ ${ }^{h}$ Department of Diagnostic and Interventional Neuroradiology, University Medical Center Hamburg-Eppendorf, Hamburg, Germany \\ 'Department of Neuroradiology, University Hospital Essen, Essen, Germany \\ 'Department of Neuroradiology, University Hospital Helsinki, Helsinki, Finland \\ kDepartment of Radiology, University Hospital Rostock, Rostock, Germany \\ 'Department of Neuroradiology, University Hospital Knappschaftskrankenhaus Bochum, Bochum, Germany \\ mInstitute of Clinical Radiology University Hospital of Muenster, Muenster, Germany \\ ${ }^{n}$ Department of Radiology, University Hospital Munich, Ludwig Maximilian University, Munich, Germany \\ 'Department of Neurology, University Hospital Helsinki, Helsinki, Finland \\ ${ }^{\mathrm{P}}$ Department of Neuroradiology, University Hospital Bonn, Bonn, Germany
}

Background and Purpose Data on safety and efficacy of intra-arterial (IA) fibrinolytics as adjunct to mechanical thrombectomy (MT) are sparse.

Methods INtra-arterial FlbriNolytics In ThrombectomY (INFINITY) is a retrospective multi-center observational registry of consecutive patients with anterior circulation large-vessel occlusion ischemic stroke treated with MT and adjunctive administration of IA fibrinolytics (alteplase [tissue plasminogen activator, tPA] or urokinase [UK]) at 10 European centers. Primary outcome was the occurrence of symptomatic intracranial hemorrhage (sICH) according to the European Cooperative Acute Stroke Study II definition. Secondary outcomes were mortality and modified Rankin Scale (mRS) scores at 3 months.

Results Of 5,612 patients screened, 311 (median age, 74 years; 44.1\% female) received additional IA after or during MT (194 MT+IA tPA, 117 MT+IA UK). IA fibrinolytics were mostly administered for rescue of thrombolysis in cerebral infarction (TICI) 0-2b after MT (80.4\%, 250/311). sICH occurred in 27 of 308 patients (8.8\%), with an increased risk in patients with initial TICIO/1 (adjusted odds
Correspondence: Johannes Kaesmacher University Institute of Diagnostic and Interventional Neuroradiology, University Hospital Bern, Inselspital, University of Bern, Freiburgstrasse 8, 3010 Bern, Switzerland

Tel: +41-31-6327325

Fax: +41-31-6329679

E-mail: Johannes.kaesmacher@insel.ch https://orcid.org/0000-0002-91772289

Received: May 14, 2020

Revised: August 15, 2020

Accepted: September 4, 2020

*These authors contributed equally to the manuscript as first author. 
ratio $[\mathrm{aOR}], 2.3 ; 95 \%$ confidence interval $[\mathrm{Cl}], 1.1$ to 5.0 per $\mathrm{TICl}$ grade decrease) or in those with intracranial internal carotid artery occlusions $(\mathrm{aOR}, 3.7 ; 95 \% \mathrm{Cl}, 1.2$ to 12.5$)$. In patients with attempted rescue of TICIO-2b and available angiographic follow-up, 116 of 228 patients (50.9\%) showed any angiographic reperfusion improvement after IA fibrinolytics, which was associated with $m R S \leq 2$ (aOR, 3.1; $95 \% \mathrm{Cl}, 1.4$ to 6.9$)$.

Conclusions Administration of IA fibrinolytics as adjunct to MT is performed rarely, but can improve reperfusion, which is associated with better outcomes. Despite a selection bias, an increased risk of sICH seems possible, which underlines the importance of careful patient selection.

Keywords Tissue plasminogen activator; Stroke; Thrombolytic therapy; Thrombectomy; Intracranial hemorrhages

\section{Introduction}

Despite recent advances in technical efficacy, incomplete and failed reperfusion results remain a significant concern and reduce the clinical benefit of mechanical thrombectomy (MT). ${ }^{1-5}$ Although the rates of thrombolysis in cerebral infarction (TICl) 3 reperfusions are constantly improving, in more than half of patients treated with MT, reperfusion is incomplete or no reperfusion is established. ${ }^{4-6}$ Potential rescue strategies consist of intracranial-stenting ${ }_{1}^{7-9}$ mechanical removal of small distal clots ${ }^{10-12}$ and administration of antiplatelets ${ }^{13}$ or fibrinolytics. ${ }^{14-16}$

The updated 2019 American Heart Association/American Stroke Association guidelines state that the use of salvage technical adjuncts, including intra-arterial (IA) thrombolysis, may be reasonable to achieve $\mathrm{mTICl}$ grade $2 \mathrm{~b} / 3$ angiographic results. ${ }^{17}$ According to a recent survey, 39\% of the responders stated to use IA recombinant tissue plasminogen activator (tPA) on individual case basis during $\mathrm{MT} .^{18}$ Observational data suggested the use of IA fibrinolytics during MT as a therapy option in selected patients, potentially improving reperfusion..$^{14-16,19}$ However, there were mixed signals regarding a potential increase in symptomatic intracranial hemorrhage $(\mathrm{sICH})$ and evidence regarding its treatment effect leading to improved reperfusion is still limited. ${ }^{14-16}$

Aim of this multi-center analysis was to report on frequency, indication, safety and efficacy of IA fibrinolytics as adjunct to mechanical thrombectomy in consecutive patients from ten European tertiary care centers.

\section{Methods}

\section{Patients}

Ten European tertiary care centers with local prospective thrombectomy databases were invited to participate in this retrospective pooled individual patient data analysis (registry name: INtra-arterial FlbriNolytics In ThrombectomY [INFINITY]). All consecutive patients treated with MT and IA administration of alteplase (referred to as tPA) or urokinase (referred to as UK) were included. Participating centers were asked to contribute all patients in whom IA fibrinolytics were administered during or after MT for an intracranial internal carotid artery (ICA), M1 or M2 occlusion. Centers were invited to report rates of MT for these occlusion sites without additional IA fibrinolytics (Table 1). Data for all patients were collected using a standardized form with predefined variables. Local investigators completed the forms systematically using data from prospectively ascertained in-hospital thrombolysis or stroke registries enhanced with additional data from patients' records and charts. Completed forms from all centers were sent to the coordinating center in Bern, where analyses of pooled data were performed.

All patients were treated with approved second-generation devices, i.e., stent-retriever or large-bore aspiration catheters or a combination thereof. Ethical approval was obtained from all local ethics committees. Consent was waived according to the retrospective nature of the work, or patients gave their written or oral consent, depending on the centers ethical and institutional guidelines. Baseline characteristics and patient demographics were extracted from the centers prospective stroke databases. Functional outcome was assessed at three months using the modified Rankin Scale (mRS). The score was evaluated by a neurologist during an outpatient visit or during a structured telephone interview by a mRS-certified nurse. Functional independence was defined as $\mathrm{mRS} \leq 2$. Day 90 functional outcome was available for 300/311 patients (96.5\%).

\section{Image analysis}

In each participating site, all images of the center's patients treated with MT and adjunctive administration of IA fibrinolytics were reevaluated by a neurointerventionalist. Indication for administering IA fibrinolytics was determined by reviewing an- 


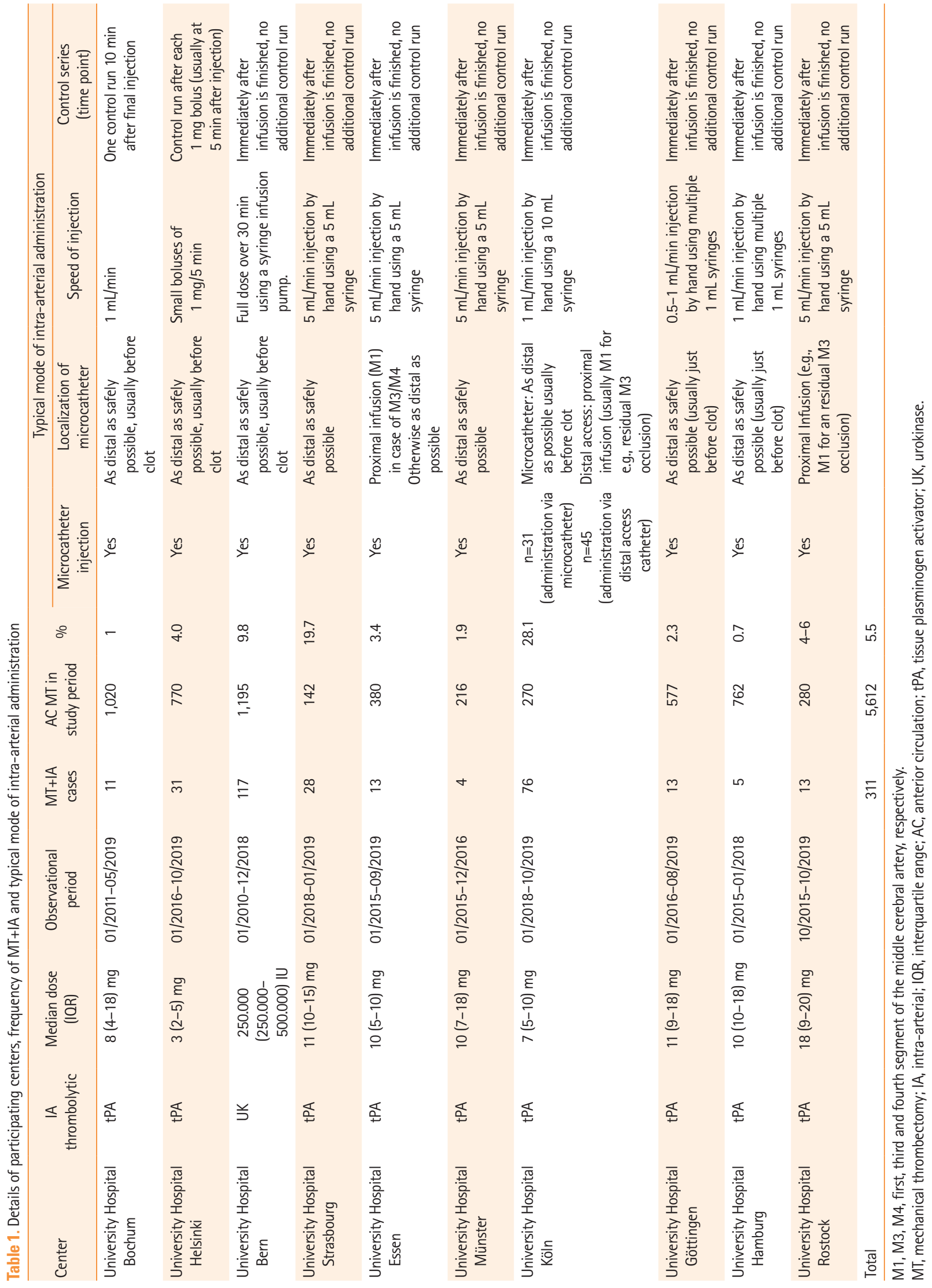


giographic images and the angiographic report. For this purpose, indications were classified into (1) rescue of TIClO-2b reperfusions after MT; (2) treatment of emboli in a new territory; or (3) administration before or during the first or second stentretriever deployment at the operator's discretion. In cases of rescue for $\mathrm{TICIO}-2 b$ reperfusions, images were assessed before and after administration of IA fibrinolytics. For this purpose, $\mathrm{TICl}$ grades before and after administration of IA fibrinolytics were documented. Moreover the occurrence of any angiographic reperfusion improvement, defined as a reduction of capillary phase deficit by newly established antegrade flow, was assessed. By definition, any angiographic reperfusion improvement could therefore include cases with and without a $\mathrm{TICl}$ grade change. This angiographic data was available in 228/250 patients with attempted rescue of TIClO-2b, because in 22 patients no angiography runs after administration of IA fibrinolytics were performed. $\mathrm{sICH}$ was defined as any intracranial hemorrhage on follow-up imaging and clinical deterioration, as evidenced by an increase in the National Institutes of Health Stroke Scale (NIHSS) score of $\geq 4$ without a mandatory confirmed causal relationship between clinical worsening and occurrence of $\mathrm{ICH}^{20}$

\section{Statistical analysis}

Data are presented as median (interquartile range [IQR]) or as number (\%). Frequency comparisons were performed using Fisher's exact test. Non-normally distributed continuous or ordinally scaled variables were compared using Whitney-Mann U test. For calculation of 95\% confidence intervals (Cls) of frequency counts, we used the methods outlined by Clopper and Pearson, ${ }^{21}$ known as the exact binomial $\mathrm{Cl}$. To evaluate an association between two variables over multiple strata we calculated a Mantel-Haenszel common odds ratio (OR) and assessed heterogeneity of ORs using a Breslow-Day test. Clinical regression models were generally adjusted for age, sex, admission NIHSS, Alberta Stroke Program Early CT Score (ASPECTS), occlusion site, symptom-onset to reperfusion intervals, and $\mathrm{TICl}$ scores (before administration of IA fibrinolytics), according to clinical importance. For a comparison regarding IA UK versus IA tPA patients, we additionally included variables displaying significant distribution imbalances between the groups. For functional outcome, we used a binary logistic regression analysis with functional independence ( $m R S \leq 2)$ as dependent variable. For an estimate regarding $\mathrm{SICH}$, a binary logistic regression model was used including the same covariates. Results are displayed as adjusted OR and corresponding 95\% $\mathrm{Cl}$. Patients with missing follow-up were excluded from the analysis regarding functional outcome or mortality. No imputation methods were performed. A sensitivity analysis of the main outcomes was performed excluding all patients treated before 2015. Statistical analyses were carried out in SPSS Statistics version 25 (IBM Co., Armonk, NY, USA) and STATA version 15.1 (StataCorp., College Station, TX, USA).

\section{Results}

Of 5,612 patients screened, 311 (median age, 74 years; 44.1\% female) received additional IA after or during MT (relative frequency 5.5\%; range across centers $0.7 \%$ to $28.1 \%$ ) (Table 1 ). Patients presented with severe symptoms (median NIHSS 14; IQR, 9 to 19) and 170 patients (54.7\%) were treated for an acute occlusion of the M1. Most commonly, IA fibrinolytics were administered via a microcatheter, which was positioned as distal as possible and usually just proximal to the residual occlusion site (Table 1). In 250 patients (80.4\%), IA fibrinolytics were administered for rescue of a $\mathrm{TICIO} / 1$ in 32 patients $(12.8 \%)$, for rescue of $\mathrm{TICl} 2 \mathrm{a}$ in 54 patients $(21.6 \%)$ or rescue of $\mathrm{TICl} 2 \mathrm{~b}$ in 164 patients $(65.6 \%)$ reperfusions after MT. Other indications were treatment for emboli in a new territory in 12 patients (3.9\%) or during first maneuvers of mechanical thrombectomy at the operator's discretion in 49 patients (15.8\%). In 117 patients (37.6\%, from one center), IA UK was administered, while 194 patients $(62.4 \%$, from nine centers) were treated with IA tPA. Median dose of IA tPA was $10 \mathrm{mg}$ (IQR, 5 to 10) and median dose of IA UK was $250.000 \mathrm{U}(I Q R, 250.000$ to 500.000). IA tPA dose tended to be higher in patients with prior IV tPA administration (median $10 \mathrm{mg}$ vs. $7.5 \mathrm{mg}, P=0.07$ ). For IA $U K$, dose regimens were higher in patients treated without prior IV tPA (median $350.000 \mathrm{U}$ vs. $250.000 \mathrm{U}, P=0.04$ ). Of 299 patients with available data, median symptom-onset to administration of IA thrombolytics was 256 minutes (IOR, 206 to 320). Correspondingly, 127 patients (42.5\%) and 43 patients (14.4\%) of patients received IA fibrinolytics beyond 4.5 and 6 hours, respectively. IA fibrinolytics were administered at a median delay of 55 minutes (IQR, 38 to 88) after groin puncture. Other baseline characteristics of the cohort and stratification by IA TPA versus IA UK can be found in Table 2.

\section{Safety evaluation}

Of 308 patients with available imaging follow-up, 27 had sICH (8.8\%; $95 \% \mathrm{Cl}, 5.9$ to 12.5 ). Rates were numerically higher in patients treated with IA tPA as compared to patients treated with IA UK (10.3\% vs. $6.1 \%, P=0.30)$. There were three missing imaging follow-ups in the UK group. Assuming a worst casescenario, the number of patients with $\mathrm{sICH}$ would have changed to $30(9.6 \% ; 95 \% \mathrm{Cl}, 6.6 \%$ to $13.5 \%)$. After adjustment for clinical confounders outlined in the methods section 
Table 2. Baseline characteristics

\begin{tabular}{|c|c|c|c|c|}
\hline Characteristic & All patients $(n=311)$ & IA tPA $(n=194)$ & IA UK $(n=117)$ & $P$ \\
\hline Age (yr) & $74(62.7-81.0)$ & $75.0(65.0-82.0)$ & $70.8(58.6-78.4)$ & 0.019 \\
\hline Female sex & $137(44.1)$ & $91(46.9)$ & 46 (39.3) & 0.197 \\
\hline Pre-stroke independence ${ }^{*}$ & $283(91.3)$ & & & 0.219 \\
\hline \multicolumn{5}{|l|}{ Risk factors } \\
\hline Atrial fibrillation ${ }^{+}$ & $144(47.2)$ & $97(50.0)$ & $47(32.6)$ & 0.233 \\
\hline Arterial hypertension & $230(74.0)$ & $144(74.2)$ & $86(73.5)$ & 0.895 \\
\hline Diabetes $^{*}$ & $73(23.6)$ & $57(29.4)$ & $16(13.9)$ & $0.002^{\S}$ \\
\hline Coronary artery disease & $110(36.4)$ & $85(45.9)$ & $25(21.4)$ & $<0.001^{\S}$ \\
\hline Smoking & $92(29.6)$ & $63(32.5)$ & $29(31.5)$ & 0.160 \\
\hline Antiplatelets & & & & 0.088 \\
\hline None & $220(71.4)$ & $146(75.6)$ & $74(64.3)$ & \\
\hline Aspirin & 79 (25.6) & $43(22.3)$ & $36(31.3)$ & \\
\hline Aspirin+clopidogrel/prasugrel & $9(2.9)$ & $4(2.1)$ & $5(4.3)$ & \\
\hline Oral anticoagulation $^{\ddagger}$ & & & & $0.049^{*}$ \\
\hline None & 259 (83.8) & 155 (80.3) & $104(89.7)$ & \\
\hline Vitamin $\mathrm{K}$ antagonists & $25(8.1)$ & $17(8.8)$ & $8(6.9)$ & \\
\hline Direct oral anticoagulants & $25(8.1)$ & 21(10.9) & $4(3.5)$ & \\
\hline Witnessed symptom-onset & $258(83.0)$ & 168 (86.6) & $90(76.9)$ & \\
\hline Symptom-onset/last-seen well to admission $(\mathrm{min})^{++}$ & $80(56-150)$ & $75(50-137)$ & $83(65-160)$ & $0.027^{* *}$ \\
\hline Admission National Institute of Health Stroke Scale & $14(9-19)$ & $15(10-18)$ & $13(8-19)$ & 0.374 \\
\hline Admission Imaging & & & & $<0.001^{\S}$ \\
\hline CT & $186(59.8)$ & $148(76.3)$ & $38(32.5)$ & \\
\hline MRI & $125(40.2)$ & $46(23.7)$ & $79(67.5)$ & \\
\hline Alberta Stroke Program Early CT Score (ASPECTS) ${ }^{\dagger}$ & $9(7-10)$ & $9(8-10)$ & $7(6-9)$ & $<0.001^{\S}$ \\
\hline ASPECTS based on non-contrast CT & $9(8-10)$ & $10(9-10)$ & $8(6-9)$ & $<0.001^{\S}$ \\
\hline ASPECTS based on MRI (diffusion-weighted imaging) & $7(6-9)$ & $8(7-9)$ & $7(5-8)$ & 0.098 \\
\hline Occlusion site & & & & 0.648 \\
\hline Intracranial ICA & $64(20.6)$ & $38(19.6)$ & $26(22.2)$ & \\
\hline First segment of the middle cerebral artery & $170(54.7)$ & $110(56.7)$ & $60(51.3)$ & \\
\hline Second segment of the middle cerebral artery & $77(24.8)$ & $46(23.7)$ & $31(26.5)$ & \\
\hline Intravenous tissue plasminogen activator & $156(50.2)$ & $107(55.2)$ & $49(41.9)$ & $0.026^{* *}$ \\
\hline Indication for IA fibrinolytics & & & & $<0.001^{\S}$ \\
\hline $\begin{array}{l}\text { Rescue thromoblysis in cerebral infarction } 0-2 b \\
\text { reperfusion }\end{array}$ & $250(80.4)$ & $168(86.6)$ & $82(70.1)$ & \\
\hline Treatment of emboli to new territory & $12(3.9)$ & $4(2.1)$ & $8(6.8)$ & \\
\hline Other/individual operator decision & 49 (15.8) & $22(11.3)$ & $27(23.1)$ & \\
\hline Symptom-onset/last-seen well to IA fibrinolytics (min) ${ }^{\ddagger \neq}$ & $256(206-320)$ & $240(189-318)$ & $275(230-324)$ & $0.012^{*}$ \\
\hline Groin puncture to IA fibrinolytics $(\min )^{\ddagger}$ & $55(38-88)$ & $48(30-75)$ & $69(48-102)$ & $<0.001^{\S}$ \\
\hline $\begin{array}{l}\text { Symptom-onset/last-seen well to reperfusion/ } \\
\text { symptom-onset/last-seen well to final angiography } \\
\text { run }(\mathrm{min})^{\S \varsigma}\end{array}$ & $268(213-350)$ & $248(200-315)$ & $206(250-381)$ & $<0.001^{\S}$ \\
\hline Final $\mathrm{TICl}$ score & & & & $0.028^{* \prime}$ \\
\hline 0 & $9(2.9)$ & $2(1.0)$ & $7(6.0)$ & \\
\hline 1 & $15(4.8)$ & $7(3.6)$ & $8(6.8)$ & \\
\hline $2 a$ & $47(15.1)$ & 26 (13.4) & 21 (17.9) & \\
\hline
\end{tabular}


Table 2. Continued

\begin{tabular}{lccc}
\hline Characteristic & All patients $(\mathrm{n}=311)$ & IA tPA (n=194) & IA UK (n=117) \\
\hline $2 b$ & $185(59.5)$ & $125(64.4)$ & $60(51.3)$ \\
3 & $55(17.7)$ & $34(17.5)$ & $21(17.9)$ \\
\hline
\end{tabular}

Values are presented as median (interquartile range) or number (\%).

$I A$, intra-arterial; tPA, tissue plasminogen activator; UK, urokinase; $C T$, computed tomography; MRI, magnetic resonance imaging; ICA, internal carotid artery; $\mathrm{TICl}$, thrombolysis in cerebral infarction.

${ }^{*}$ Data available for $310 ;{ }^{+}$Data available for $305 ;{ }^{*}$ Data available for $309 ;{ }^{5} P<0.01$; "Data available for $302 ;$ ' Data available for $308 ;{ }^{* *} P<0.05$; ${ }^{+t}$ Data available for 303; ${ }^{* \dagger}$ Data available for 299; ${ }^{\S \S}$ Data available for 295.

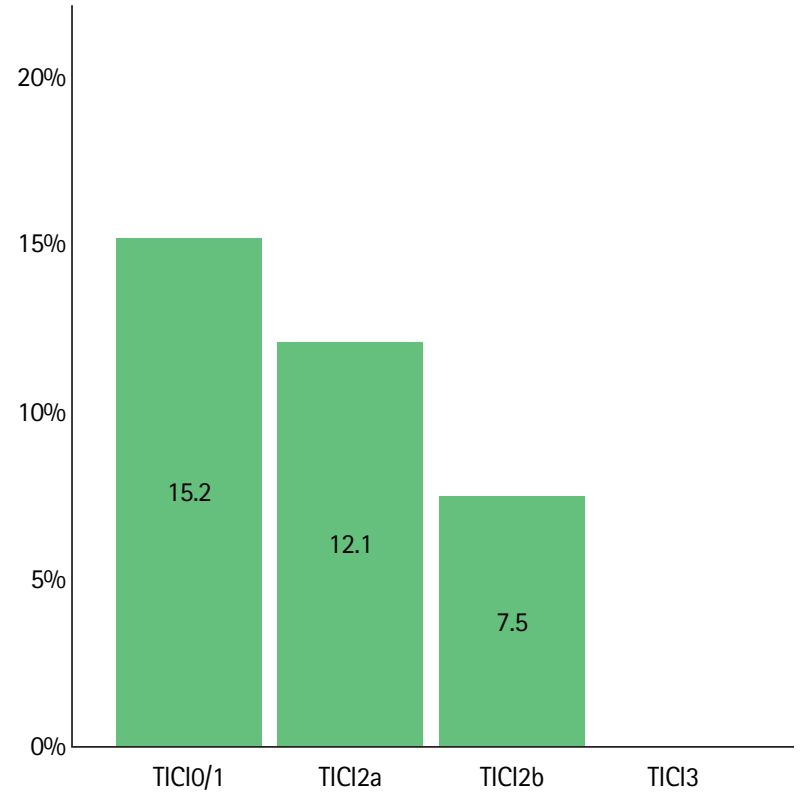

Figure 1. Risk of symptomatic intracranial hemorrhage (sICH) according to thrombolysis in cerebral infarction (TICI). Data on $\mathrm{SICH}$ was available in $308 / 311$ patients. SICH occurred in 5/33 patients (15.2\%) with $\mathrm{TIClO} / 1$, in $8 / 66$ patients $(12.1 \%)$ with $\mathrm{TICl} 2 \mathrm{a}$, in $14 / 187$ patients $(7.5 \%)$ with $\mathrm{TICl} 2 \mathrm{~b}$ and did not occur in 22 patients with $\mathrm{TICl} 3$. There was a decreased risk of sICH with higher $\mathrm{TICl}$ grade (adjusted odds ratio [aOR] per grade increase derived from logistic regression analysis: $\mathrm{aOR}, 0.43 ; 95 \%$ confidence interval, 0.20 to 0.94$)$. $\mathrm{TICl}$ scores used were before administration of intra-arterial fibrinolytics in cases of rescue of $\mathrm{TIClO}-2 b$ reperfusions. In other cases (treatment of emboli in new territory or administration during first retrievals at the operator's discretion) final $\mathrm{TICl}$ scores were used.

and imbalances in baseline variables between the fibrinolytics groups, the point estimate favored IA UK with a trend for fewer sICH (adjusted odds ratio [aOR], $0.53 ; 95 \% \mathrm{Cl}, 0.27$ to 1.04 ) (Supplementary Table 1 for other regression coefficients). In the model, there was also an association of female sex $(\mathrm{aOR}$ $0.30 ; 95 \% \mathrm{Cl}, 0.095$ to 0.97 ) and $\mathrm{M} 1 / \mathrm{M} 2$ occlusions ([aOR, $0.27 ; 95 \% \mathrm{Cl}, 0.08$ to 0.86 ] and $[\mathrm{aOR}, 0.29 ; 95 \% \mathrm{Cl}, 0.07$ to 1.14], respectively) with lower rates of sICH. Moreover, $\mathrm{TICl}$ grades before administration of IA fibrinolytics were associated with $\mathrm{SICH}$, with a lower risk observed in better $\mathrm{TICl}$ grades (aOR, $0.43 ; 95 \% \mathrm{Cl}, 0.20$ to 0.94 for $\mathrm{TICl}$ grade increase) (Figure
1). The estimates also pointed towards an increased risk of $\mathrm{sICH}$ in patients with pretreatment with IV tPA $(\mathrm{aOR}, 2.33$; $95 \% \mathrm{Cl}, 0.75$ to 7.17$)$, prior use of Aspirin $(\mathrm{aOR}, 2.30 ; 95 \% \mathrm{Cl}$, 0.74 to 7.09 , comparator no antiplatelet medication) and those taking direct oral anticoagulants $(\mathrm{aOR}, 3.08 ; 95 \% \mathrm{Cl}, 0.55$ to 17.28 , comparator no oral anticoagulant medication); however, these associations did not reach statistical significance. Raw group comparisons of sICH occurrence with strata of antiplatelets and intake of oral anticoagulants can be found in Supplementary Table 2. Doses of IA tPA/UK did not differ between patients with and without sICH (median $10 \mathrm{mg}$ vs. $10 \mathrm{mg}, P=0.96$; and $250,000 \mathrm{IU}$ vs. $250,000 \mathrm{IU}, P=0.48$ ).

After 3 months, 50 of 300 patients (16.7\%) with available follow-up died, with a doubled mortality rate in patients with sICH (eight of $26 \mathrm{sICH}$ patients with available follow-up, 30.8\%).

\section{Angiographic efficacy}

Angiographic control runs after administration of IA fibrinolytics were available, for 228 of 250 patients (91.2\%), who received IA fibrinolytics for attempted rescue of TIClO-2b reperfusions after MT. In 116 of those 228 (50.9\%), any angiographic reperfusion improvement was noted, which resulted in a $\mathrm{TICl}$ grade improvement in 66 of 228 patients (28.9\%) (Figure 2). Occurrence of any angiographic reperfusion improvement did not differ according to $\mathrm{TICl}$ strata before administration of IA fibrinolytics or occlusion sites (Supplementary Table 3). In 11 patients, in whom IA fibrinolytics were administered for treatment of an embolus in a new territory, successful reperfusion was reported in six patients (54.5\%). In 49 patients, in whom IA fibrinolytics were administered before or during the first thrombectomy maneuvers at the operator's discretion, final $\mathrm{TICl}$ grades were $\mathrm{TICl} 3$ in 16 patients (32.7\%), $\mathrm{TICl} 2 \mathrm{~b}$ in $17 \mathrm{pa}-$ tients (34.7\%), $\mathrm{TICl} 2 \mathrm{a}$ in 13 patients (26.5\%), and $\mathrm{TIClO} / 1$ in three patients (6.1\%).

\section{Functional outcome}

At 3 months follow-up, 111 of 300 patients (37.0\%) achieved 


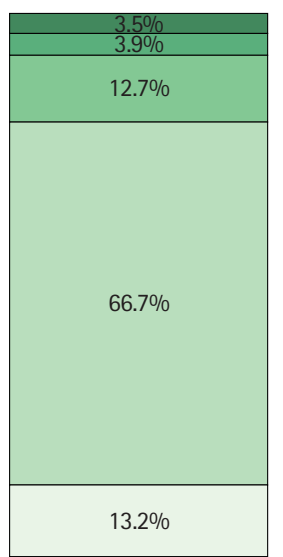

After IA fibrinolytics

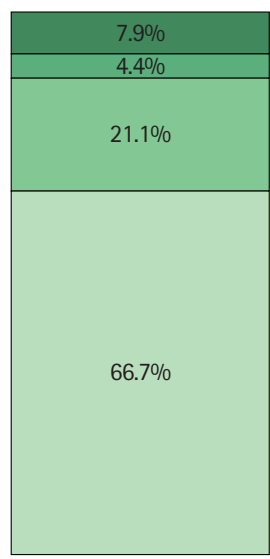

Before IA fibrinolytics

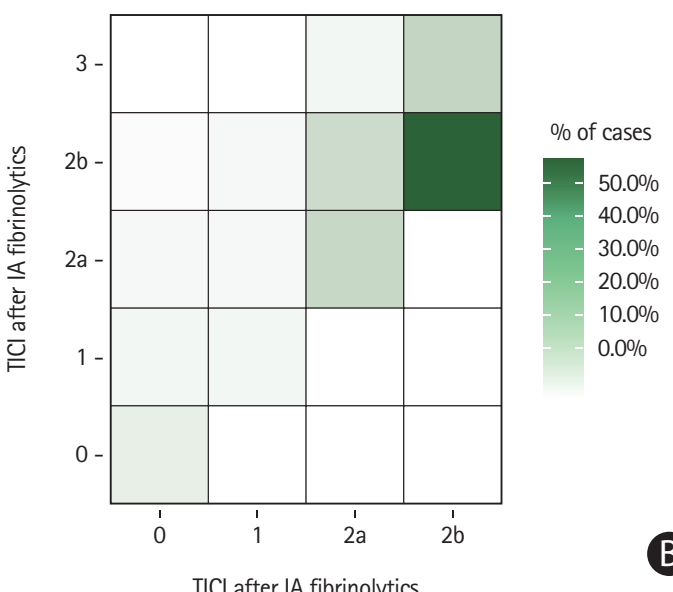

B

Figure 2. Thrombolysis in cerebral infarction (TICI) grade change after intra-arterial (IA) fibrinolytics. (A) After IA fibrinolytics, TICl shifted towards better scores ( $\mathrm{TICl}$ grade improvement noted in $28.9 \%$ of patients). (B) Most patients with $\mathrm{TICl} 2 \mathrm{~b}$ did not improve to $\mathrm{TICl} 3$; however, any angiographic reperfusion improvement was relatively common in patients with initial $\mathrm{TICl} 2 \mathrm{~b}$ reperfusions $(46.1 \%, 70 / 152)$.

300 Complete cohort

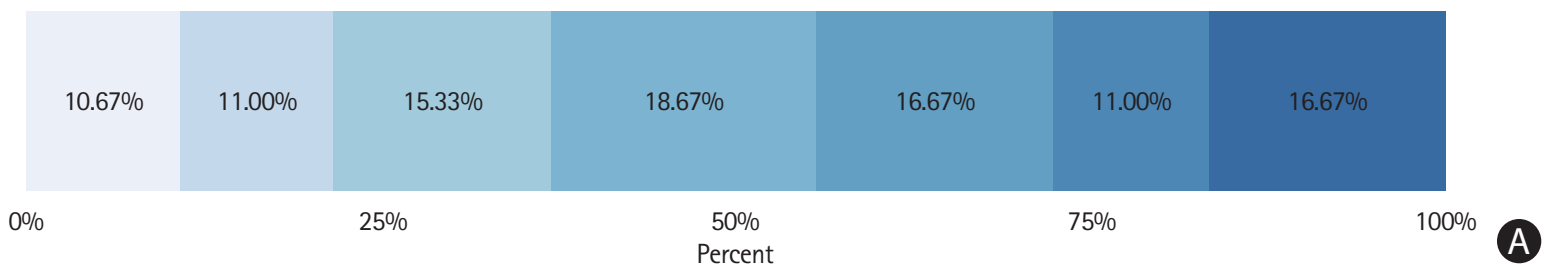

187 IA tPA cohort

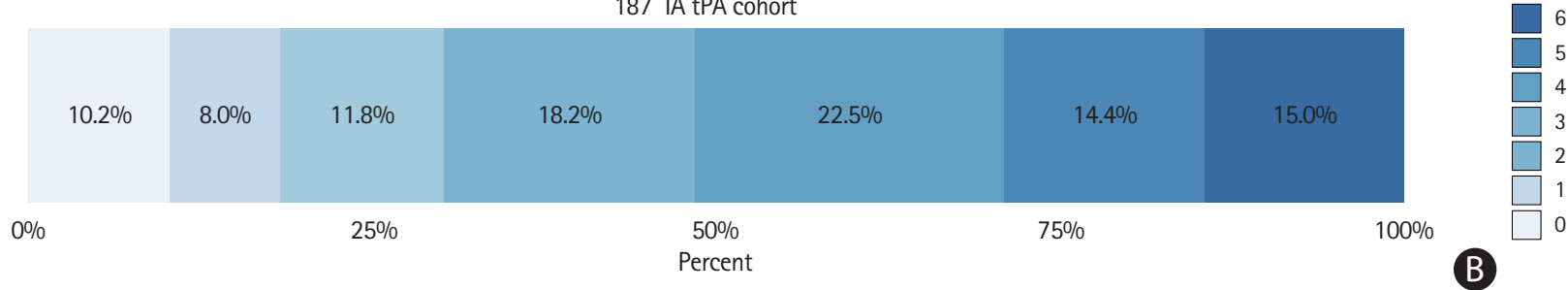

113 IA UK cohort

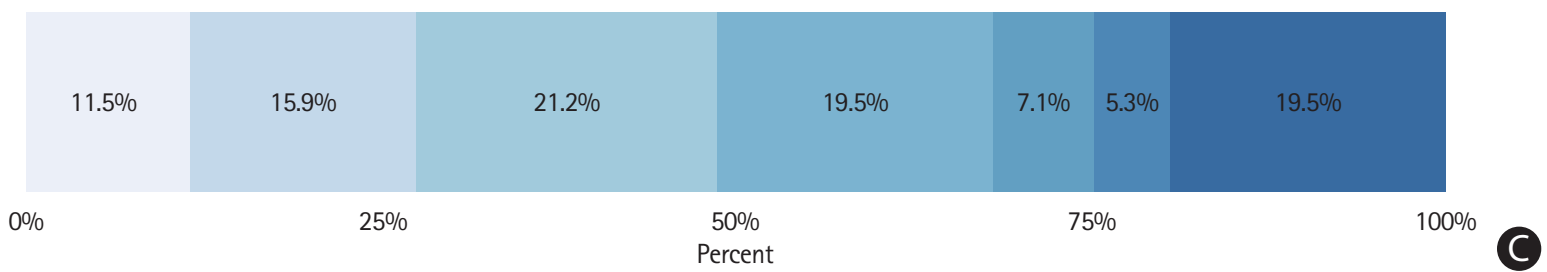

Figure 3. Three-month functional outcome of patients treated with mechanical thrombectomy (MT) and intra-arterial (IA) fibrinolytics. (A) Three-month functional outcome was available for 300/311 patients treated with MT+IA fibrinolytics. Functional independence (modified Rankin Scale [mRS] $\leq 2$ ) was observed in 37.0\% (111/300) of patients and 16.7\% (50/300) of patients had died. (B, C) Functional outcomes were better in patients receiving IA urokinase (UK) (odds ratio for $m R S \leq 2,2.22 ; 95 \%$ confidence interval [Cl], 1.37 to 3.60). However, this association was not statistical significant after adjustment for clinical confounders and baseline group imbalances (IA UK vs. IA tissue plasminogen activator [tPA]: adjusted odds ratio, 1.35; $95 \%$ Cl, 0.87 to 2.09).

$m R S \leq 2$ (Figure 3A). Functional outcomes were better in patients receiving IA UK (OR for $m R S \leq 2,2.22 ; 95 \% \mathrm{Cl}, 1.37$ to 3.60) (Figure 3B and C). However, this association was not sta- tistically significant after adjustment for clinical confounders and baseline group imbalances (IA UK vs. IA tPA: adjusted OR, $1.35 ; 95 \% \mathrm{Cl}, 0.87$ to 2.09 ). Significant variables associated 
110 Angiographic reperfusion improvement after additional IA fibrinolytics

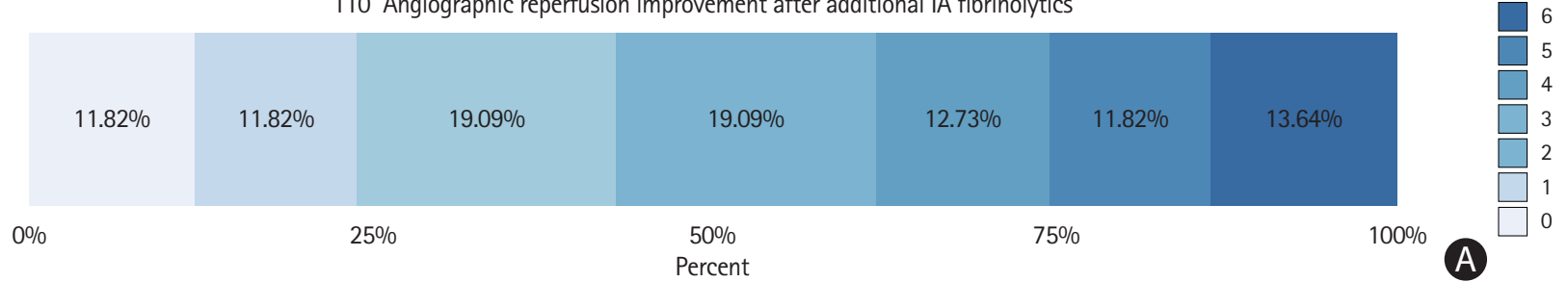

111 No angiographic reperfusion improvement after additional IA fibrinolytics

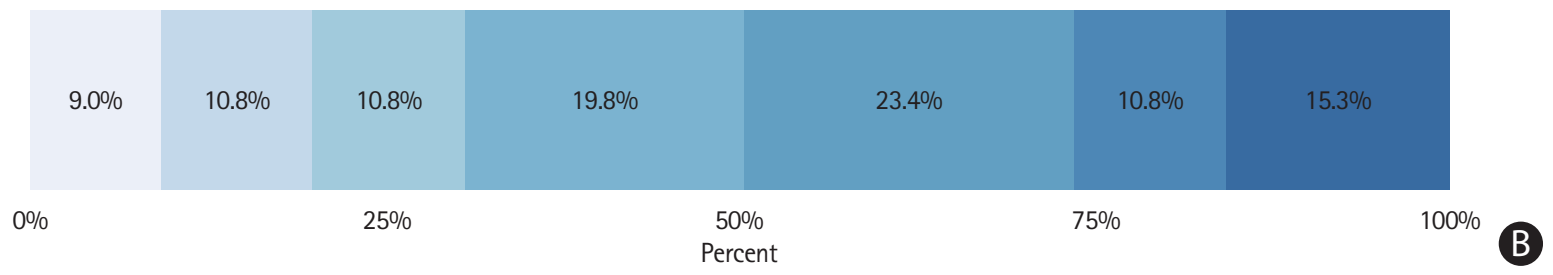

Figure 4. Three-month functional outcome with strata of angiographic reperfusion improvement after administering intra-arterial (IA) fibrinolytics. For 228/250 patients with an intention to improve TICl 0-2b, angiographic control runs after administration of IA fibrinolytics were available. Three-month functional outcome was available in 221 of these 228 patients. (A) Any angiographic improvement was observed in 110/221 and was associated with modified Rankin Scale (mRS) $\leq 2$ after adjusting for covariates outlined in the methods section (adjusted odds ratio, 3.11; 95\% confidence interval, 1.41 to 6.86 ). (B) The $111 / 221$ patients showed no angiographic improvement. $\mathrm{TICl}$, thrombolysis in cerebral infarction.

Table 3. Clinical benefit of angiographic reperfusion with strata of TICl grade before administration of IA fibrinolysis

\begin{tabular}{|c|c|c|c|c|}
\hline \multirow{2}{*}{ TICI before IA fibrinolytics } & & \multicolumn{2}{|c|}{$\mathrm{mRS}$} & \multirow{2}{*}{$\mathrm{OR}$} \\
\hline & & $>2$ & $\leq 2$ & \\
\hline \multirow[t]{3}{*}{0} & ARI- & $8(100)$ & $0(0)$ & \\
\hline & $\mathrm{ARI}+$ & $6(66.7)$ & $3(33.3)$ & \\
\hline & Total (TIClO) & $14(82.4)$ & $3(17.6)$ & $1.50(0.95-2.38)$ \\
\hline \multirow[t]{3}{*}{1} & ARI- & $3(75)$ & $1(25)$ & \\
\hline & ARI+ & $4(66.7)$ & $2(33.3)$ & \\
\hline & Total (TICl1) & $7(70)$ & $3(30)$ & $1.50(0.09-25.39)$ \\
\hline \multirow[t]{3}{*}{$2 a$} & ARI- & $14(77.8)$ & $4(22.2)$ & \\
\hline & $A R I_{+}$ & $17(63.0)$ & $10(37.0)$ & \\
\hline & Total (TICl2a) & $31(68.9)$ & $14(31.1)$ & $2.06(0.53-8.00)$ \\
\hline \multirow[t]{3}{*}{$2 b$} & ARI- & $52(64.2)$ & $29(35.8)$ & \\
\hline & $A R I_{+}$ & $36(52.9)$ & $32(47.1)$ & \\
\hline & Total (TICl2b) & $88(59.1)$ & $61(40.9)$ & $1.59(0.83-3.08)$ \\
\hline \multirow[t]{3}{*}{ All $\mathrm{TICl}$} & ARI- & $77(69.4)$ & $34(30.6)$ & \\
\hline & $\mathrm{ARI}+$ & $63(57.3)$ & $47(42.7)$ & \\
\hline & Total (all TICl) & $140(63.3)$ & 81 (36.7) & cOR $1.83(1.04-3.22)^{*}$ \\
\hline
\end{tabular}

Values are presented as number (\%). Calculated using Mantel-Haenszel statistics. P for heterogeneity of OR, 0.54 (Breslow-Day test). 95\% Confidence intervals of OR were calculated using Woolf's approximation.

$\mathrm{TICl}$, thrombolysis in cerebral infarction; IA, intra-arterial; mRS modified Rankin Scale; OR, odds ratio; ARI-/+, angiographic reperfusion improvement; cOR, common odds ratio.

${ }^{*} P<0.05$.

with $m R S \leq 2$ in the model were: age, admission NIHSS, TICl before administration of IA fibrinolytics, diabetes, coronary artery disease, and M2 occlusions (Supplementary Table 4 for point estimates and 95\% $\mathrm{Cls}$ ).

In patients with rescue of $\mathrm{TIClO}-2 \mathrm{~b}$ reperfusions after MT and available angiographic follow-up (228 patients), any angio- 
graphic reperfusion improvement was associated with $\mathrm{mRS} \leq 2$ $(\mathrm{aOR}, 3.11 ; 95 \% \mathrm{Cl}, 1.41$ to 6.86$)$ (Figure 4) after adjusting for covariates outlined in the methods section. This association of functional independence and angiographic reperfusion improvement was relatively stable across strata of reperfusion grade achieved before administering IA fibrinolytics (common $\mathrm{OR}, 1.83 ; 95 \% \mathrm{Cl}, 1.04$ to $3.22, P$ for heterogeneity of ORs, 0.54) (Table 3).

\section{Sensitivity analysis}

Excluding patients treated before 2015 lead to a subcohort of 240 patients (51 patients treated with IA UK and 189 treated with IA tPA). In this subcohort, 23 of 239 with available data had sICH (9.6\%). Of 240 patients, angiographic control runs after administration of IA fibrinolytics were available for 181 of 203 patients (89.2\%), who received IA fibrinolytics for attempted rescue of TIClO-2b reperfusions after MT. In these 181 patients, any angiographic reperfusion improvement was noted in 87 (48.1\%). The association of angiographic reperfusion improvement with $\mathrm{mRS} \leq 2$ remained comparable to the complete cohort (aOR, 5.15; 95\% $\mathrm{Cl}, 1.91$ to 13.93).

\section{Discussion}

This study has the following main findings. (1) Substantial heterogeneity regarding the frequency, dose and indications for IA fibrinolytics during or after MT can be observed across different centers. (2) In a considerable proportion of patients, IA fibrinolytics were administered after 4.5 hours, but the majority (>80\%) of patients received IA fibrinolytics within 6 hours after symptom-onset. (3) Rates of sICH were twice as high compared to rates of sICH reported in the Highly Effective Reperfusion Using Multiple Endovascular Devices (HERMES) collaboration or the Efficacy and safety of nerinetide for the treatment of acute ischaemic stroke (ESCAPE-NA1) trial (8.8\% vs. 3.9\%/4.4\%), ${ }^{6,22}$ particularly after rescue of low $\mathrm{TICl}$ scores or treatment of intracranial ICA occlusions. (4) Any angiographic reperfusion improvement was observed in half of the patients with around one-third resulting in a $\mathrm{TICl}$ grade change. (5) Any angiographic reperfusion improvement after IA fibrinolytics was associated with better outcomes.

\section{Frequency and indications}

Depending on the survey, $39 \%$ and $60.6 \%$ of survey respondents stated using IA thrombolytics in contemporary MT practice. $^{18,23}$ In our multi-center analysis, the overall rate of IA fibrinolytics administrations was only 5.5\% of all anterior circulation large-vessel occlusion MT cases, corroborating the survey's finding that around $50 \%$ of interventionalists only treat 1 to 5 cases/year with IA rtPA. ${ }^{23}$ However, considerable heterogeneity in IA fibrinolytic frequency was noted across centers, with some centers utilizing IA tPA in every fourth patient undergoing MT. Median dose of IA tPA was $10 \mathrm{mg}$, which is within the range of previous observational reports ${ }^{15}$ and dosage regimens applied in the THRombectomie des Artères CErebrales (THRACE) trial (mean dose, $8.8 \mathrm{mg}$ ). ${ }^{24}$ In line with the Prolyse in Acute Cerebral Thromboembolism II (PROACT-II) inclusion criteria, most patients were treated within 6 hours after symptom-onset, but a considerable proportion of patients was treated beyond 4.5 hours. ${ }^{25}$ Treatment outside of IV tPA eligibility criteria was also evident considering the inclusion of very low ASPECTS cases $(n=19$, ASPECTS $<5)$ and treatment of patients with prior anticoagulation (16\%).

\section{Risk of bleeding}

sICH has always been a major concern in patients treated with IA fibrinolytics. Meta-analysis of randomized-controlled trials (RCTs) evaluating IA fibrinolytics as stand-alone approach observed a $6.6 \%$ excess risk of sICH in patients treated with IA fibrinolytics as compared to controls (OR, 2.87; $8.9 \%$ vs. $2.3 \%$, number needed to harm 15). ${ }^{26}$ However, no excess in mortality was found (20.5\% vs. $24.0 \%$ ). ${ }^{26}$ In this multi-center single-arm observational cohort, we found an overall sICH rate of $8.8 \%$ (95\% Cl, 5.9 to 12.5). This compares unfavorable to rates of sICH reported in HERMES (4.4\%) ${ }^{22}$ and ESCAPE-NA1 (3.9\%). ${ }^{6}$ However, there are many constraints associated with a direct comparison. First, this observational cohort included patients with pre-stroke disability, patients under oral anticoagulation and lower ASPECTS as compared to recent RCTs. Second, and maybe most importantly, there was a severe selection bias towards poor reperfusion grades and hence, lengthy procedure, because an incomplete reperfusion status was the main indication for administering IA fibrinolytics. Patients in whom no reperfusion or only incomplete reperfusion can be achieved have significantly higher rates of $\mathrm{sICH}_{1}^{27-29}$ and this association was also observed in our data.

Nevertheless, known excess risk from IA fibrinolytics RCTs and the possibility of an up to tripled sICH risk in this study (considering the upper 95\% Cl reported here) should prompt very careful patient selection. This comprises identification of patients with an a priori high risk of $\mathrm{sICH}$, including patients with initial TICIO/1 and intracranial ICA occlusion as observed in our cohort. Also other, more general factors associated with sICH in other MT cohorts like history of diabetes, high blood pressure, age, high NIHSS, low ASPECTS, and poor collaterals should be acknowledged. ${ }^{27,29}$ The lack of a statistically signifi- 
cant association of sICH and prior IV tPA, Aspirin or anticoagulant intake should not be mistaken for clear safety signals, as the number of patients included in these subgroups were small, uncertainty is high and the point estimates were directed towards an increased risk. We did not observe a clear association of dose-risk effects, which has been reported by others. ${ }^{30} \mathrm{How}$ ever, as dose-regimens were not randomized, we cannot exclude that doses applied were modified according to the patients presumed risk of bleeding, including pretreatment with IV tPA.

Surprisingly, the rate of sICH appeared lower after IA UK as compared to IA tPA, which seemed tangible after adjustment for clinical confounders and baseline imbalances between the groups. There is some preclinical evidence that UK upregulates blood brain barrier tight junctions and has a more favorable effect on matrix metalloproteinases, potentially lowering the risk of bleeding in comparison to tPA. ${ }^{31,32}$ However, we want to stress that this result was not statistically significant and should be interpreted cautiously. Patients treated with IA UK were treated at one center only, while IA tPA patients were treated at nine different European centers. While we have tried to account for baseline differences between the groups, there is considerable room for hidden bias reflecting the centers individual decisions and practice to select patients for MT+IA fibrinolytic treatments.

\section{Angiographic reperfusion improvement}

Any angiographic reperfusion improvement was observed in approximately 50\% of patients. These results corroborate previous observations, showing that administration of IA fibrinolytics improved reperfusion after failed MT. ${ }^{14}$ Notably, angiographic control runs were usually performed immediately after termination of the IA fibrinolytics infusion. Despite this promising observation and plausible causality, there is some uncertainty regarding the degree of this observation caused by IA fibrinolytics. Theoretically some of the observed residual capillary perfusion deficits may also dissolve spontaneously (i.e., without additional administration of IA fibrinolytics), if angiographic control runs would have been performed with a considerable delay in patients without additional administration of IA fibrinolytics.

On the other hand, we were unable to evaluate delayed reperfusion after administration of IA fibrinolytics and hence, the observed efficacy may seem artificially low. Despite a confinement to the immediate post-infusion period, however, angiographic reperfusion improvement was associated with superior functional outcomes and a 12.1\% absolute increase of mRS $\leq 2$, which underlines the notion that even small reperfusion improvements may be clinically significant.

\section{Outlook}

Almost half of the interventionalists believe that IA thrombolysis may have a role in modern endovascular practice, but current evidence is still limited. ${ }^{23}$ Currently, there are two ongoing trials evaluating IA fibrinolytics as adjunct to MT. The Boosting REcanalization of Thrombectomy for Ischemic Stroke by Intraarterial TNK (BRETIS-TNK) pilot study (clinicaltrials.gov, NCT04202458) will assess the safety and efficacy of IA tenecteplase continuously administered after the first attempt of a thrombectomy device pass. The CHemical OptImization of Cerebral Embolectomy (CHOICE) trial will randomize patients with $\mathrm{TICl} 2 \mathrm{~b}$ reperfusion to receive either a 30-minute IA infusion of weight-adapted tPA or IA placebo. ${ }^{33}$ The present study suggests that although $\mathrm{TICl}$ grade improvement occurs only in one-third of patients, even small non- $\mathrm{TICl}$ grade relevant angiographic perfusion improvements are associated with favorable outcomes. Because in our single-arm cohort we could not exclude a potentially up to tripled risk of $\mathrm{sICH}$, future observational studies should compare patients with MT+IA to patients with MT and failed or incomplete reperfusion without additional rescue IA fibrinolytics. Such studies may also incorporate an assessment of delayed reperfusion, which's rates remain unknown from currently available analyses.

\section{Limitations}

This is a retrospective single-arm observational study with its associated limitations. First, angiographic efficacy analyses were performed at each center independently, giving rise to inter-rater variability. Second, generalizability of the findings is limited, because the IA+MT cohort constitutes only $5.5 \%$ of all patients treated for anterior circulation large vessel occlusion at the ten participating centers. Hence, there is a very high chance of selection bias and rates of $\mathrm{sICH}$ may be even higher in an unselected cohort. Third, eleven patients (3.5\%) were lost to follow-up regarding functional outcomes, leading to attrition bias. Finally, comparison of rates of sICH with RCT trial data is limited by differences in baseline patient characteristics and a selection bias towards poor reperfusion grades.

\section{Conclusions}

Administration of IA fibrinolytics as adjunct to MT is performed rarely, but can improve reperfusion, which is associated with better outcomes. Even when considering a selection bias favoring patients with poor reperfusion, an excess risk of sICH seems possible, which should prompt careful patient selection, partic- 
ularly in patients with intracranial ICA occlusions or attempted rescue of a $\mathrm{TIClO} / 1$ reperfusion. Potential differences regarding the rates of sICH between IA UK and IA tPA after MT warrant further studies.

\section{Supplementary materials}

Supplementary materials related to this article can be found online at https://doi.org/10.5853/jos.2020.01788.

\section{Disclosure}

Johannes Kaesmacher reports grants by the Bangerter-RhynerFoundation and the Swiss Academy of Medical Sciences related to the project. Urs Fischer reports grants from Medtronic during the conduct of the study, grants and other from Medtronic, and other from Medtronic, Stryker, and CSL Behring outside the submitted work. Jan Gralla reports grants from Medtronic and other from Penumbra outside the submitted work. All other authors report no conflict of interests.

\section{Acknowledgments}

The research was supported by the Bangerter-Rhyner-Foundation and the Swiss Academy of Medical Sciences (Johannes Kaesmacher, grant paid to institution). The funder did not take part in conceptualization, drafting or approval of the manuscript.

\section{References}

1. Rizvi A, Seyedsaadat SM, Murad MH, Brinjikji W, Fitzgerald ST, Kadirvel R, et al. Redefining 'success': a systematic review and meta-analysis comparing outcomes between incomplete and complete revascularization. J Neurointerv Surg 2019;11: 9-13.

2. Liebeskind DS, Bracard $S$, Guillemin F, Jahan R, Jovin TG, Majoie $C B$, et al. eTICl reperfusion: defining success in endovascular stroke therapy. J Neurointerv Surg 2019;11:433-438.

3. Kaesmacher J, Dobrocky T, Heldner MR, Bellwald S, Mosi-

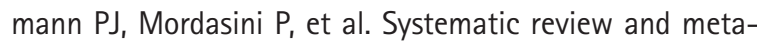
analysis on outcome differences among patients with $\mathrm{TICl} 2 \mathrm{~b}$ versus TICl3 reperfusions: success revisited. J Neurol Neurosurg Psychiatry 2018;89:910-917.

4. Kaesmacher J, Gralla J, Mosimann PJ, Zibold F, Heldner MR, Piechowiak $E_{1}$ et al. Reasons for reperfusion failures in stentretriever-based thrombectomy: registry analysis and proposal of a classification system. AJNR Am J Neuroradiol 2018;39:
1848-1853.

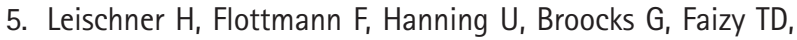
Deb-Chatterji $\mathrm{M}$, et al. Reasons for failed endovascular recanalization attempts in stroke patients. J Neurointerv Surg 2019;11:439-442.

6. Hill MD, Goyal M, Menon BK, Nogueira RG, McTaggart RA, Demchuk AM, et al. Efficacy and safety of nerinetide for the treatment of acute ischaemic stroke (ESCAPE-NA1): a multicentre, double-blind, randomised controlled trial. Lancet 2020:395:878-887.

7. Baek JH, Kim BM, Kim DJ, Heo JH, Nam HS, Yoo J. Stenting as a rescue treatment after failure of mechanical thrombectomy for anterior circulation large artery occlusion. Stroke 2016;47:2360-2363.

8. Meyer L, Fiehler J, Thomalla G, Krause LU, Lowens S, Rothaupt J, et al. Intracranial stenting after failed thrombectomy in patients with moderately severe stroke: a multicenter cohort study. Front Neurol 2020;11:97.

9. Chang Y, Kim BM, Bang OY, Baek JH, Heo JH, Nam HS, et al. Rescue stenting for failed mechanical thrombectomy in acute ischemic stroke: a multicenter experience. Stroke 2018:49:958-964.

10. Grossberg JA, Rebello LC, Haussen DC, Bouslama M, Bowen $M$, Barreira $C M$, et al. Beyond large vessel occlusion strokes: distal occlusion thrombectomy. Stroke 2018:49:1662-1668.

11. Goyal M, Ospel JM, Menon BK, Hill MD. MeVO: the next frontier? J Neurointerv Surg 2020;12:545-547.

12. Kaesmacher J, Maegerlein $C_{1}$ Zibold $F_{1}$ Wunderlich S, Zimmer $C_{1}$ Friedrich B. Improving $\mathrm{mTICl} 2 \mathrm{~b}$ reperfusion to $\mathrm{mTICl} 2 \mathrm{c} / 3$ reperfusions: a retrospective observational study assessing technical feasibility, safety and clinical efficacy. Eur Radiol 2018;28:274-282

13. Yang $M$, Huo $X$, Gao F, Wang $A$, Ma N, Shi $H$, et al. Low-dose rescue tirofiban in mechanical thrombectomy for acute cerebral large-artery occlusion. Eur J Neurol 2020;27:1056-1061.

14. Zaidi $S F$, Castonguay $A C$, Jumaa MA, Malisch TW, Linfante I, Marden FA, et al. Intraarterial thrombolysis as rescue therapy for large vessel occlusions. Stroke 2019;50:1003-1006.

15. Anadani M, Ajinkya S, Alawieh A, Vargas J, Chatterjee A, Turk $A$, et al. Intra-arterial tissue plasminogen activator is a safe rescue therapy with mechanical thrombectomy. World Neurosurg 2019;123:e604-e608.

16. Kaesmacher J, Bellwald S, Dobrocky T, Meinel TR, Piechowiak $\mathrm{El}$, Goeldlin $\mathrm{M}$, et al. Safety and efficacy of intra-arterial urokinase after failed, unsuccessful, or incomplete mechanical thrombectomy in anterior circulation large-vessel occlusion stroke. JAMA Neurol 2020;77:318-326.

17. Powers WJ, Rabinstein AA, Ackerson T, Adeoye OM, Bam- 
bakidis NC, Becker K, et al. Guidelines for the early management of patients with acute ischemic stroke: 2019 update to the 2018 guidelines for the early management of acute ischemic stroke: a guideline for healthcare professionals from the American Heart Association/American Stroke Association. Stroke 2019;50:e344-e418.

18. Kellert $\mathrm{L}$, Wollenweber FA, Thomalla G, Nolte $\mathrm{CH}$, Fiehler J, Ringleb PA, et al. Thrombolysis management in thrombectomy patients: real-life data from German stroke centres. Eur Stroke J 2017;2:356-360.

19. Heiferman DM, Li DD, Pecoraro NC, Smolenski AM, Tsimpas A, Ashley WW Jr. Intra-arterial alteplase thrombolysis during mechanical thrombectomy for acute ischemic stroke. $J$ Stroke Cerebrovasc Dis 2017;26:3004-3008.

20. Hacke W, Kaste M, Fieschi C, von Kummer R, Davalos A, Meier $D$, et al. Randomised double-blind placebo-controlled trial of thrombolytic therapy with intravenous alteplase in acute ischaemic stroke (ECASS II). Second European-Australasian Acute Stroke Study Investigators. Lancet 1998;352:12451251.

21. Clopper CJ, Pearson ES. The use of confidence or fiducial limits illustrated in the case of the binomial. Biometrika 1934; 26:404-413.

22. Goyal M, Menon BK, van Zwam WH, Dippel DW, Mitchell PJ, Demchuk AM, et al. Endovascular thrombectomy after largevessel ischaemic stroke: a meta-analysis of individual patient data from five randomised trials. Lancet 2016;387:17231731.

23. Castonguay AC, Jumaa MA, Zaidat 00, Haussen DC, Jadhav

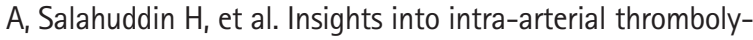
sis in the modern era of mechanical thrombectomy. Front Neurol 2019;10:1195.

24. Bracard S, Ducrocq $X$, Mas JL, Soudant M, Oppenheim C,

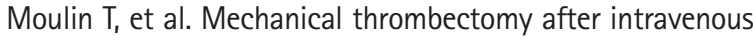
alteplase versus alteplase alone after stroke (THRACE): a ran- domised controlled trial. Lancet Neurol 2016;15:1138-1147.

25. Furlan A, Higashida R, Wechsler L, Gent M, Rowley H, Kase C, et al. Intra-arterial prourokinase for acute ischemic stroke. The PROACT II study: a randomized controlled trial. Prolyse in Acute Cerebral Thromboembolism. JAMA 1999;282:20032011.

26. Lee $M$, Hong KS, Saver JL. Efficacy of intra-arterial fibrinolysis for acute ischemic stroke: meta-analysis of randomized controlled trials. Stroke 2010;41:932-937.

27. Cappellari M, Mangiafico S, Saia V, Pracucci G, Nappini S, Nencini $\mathrm{P}$, et al. IER-SICH nomogram to predict symptomatic intracerebral hemorrhage after thrombectomy for stroke. Stroke 2019;50:909-916.

28. Lee YB, Yoon W, Lee YY, Kim SK, Baek BH, Kim JT, et al. Predictors and impact of hemorrhagic transformations after endovascular thrombectomy in patients with acute large vessel occlusions. J Neurointerv Surg 2019;11:469-473.

29. Kaesmacher J, Kaesmacher M, Maegerlein C, Zimmer C, Gersing AS, Wunderlich $S$, et al. Hemorrhagic transformations after thrombectomy: risk factors and clinical relevance. Cerebrovasc Dis 2017;43:294-304.

30. Hassan AE, Abd-Allah F, Chaudhry SA, Adil MM, Rostambeigi $\mathrm{N}$, Qureshi Al. A critical analysis of intra-arterial thrombolytic doses in acute ischemic stroke treatment. Neurocrit Care 2014;21:119-123.

31. Kadir RRA, Bayraktutan U. Urokinase plasminogen activator: a potential thrombolytic agent for ischaemic stroke. Cell Mol Neurobiol 2020;40:347-355

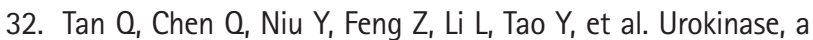
promising candidate for fibrinolytic therapy for intracerebral hemorrhage. J Neurosurg 2017;126:548-557.

33. Renú $A$, Blasco J, Millán $M$, Martí-Fàbregas J, Cardona $P$,

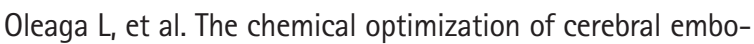
lectomy trial: study protocol. Int J Stroke 2019 Dec 18 [Epub]. https://doi.org/10.1177/1747493019895656. 
Supplementary Table 1. Logistic regression model (dependent variable symptomatic intracranial hemorrhage)

\begin{tabular}{|c|c|c|c|}
\hline Variable & $\mathrm{aOR}$ & $95 \% \mathrm{Cl}$ & $P$ \\
\hline IA UK vs. IA tPA & 0.53 & $0.27-1.04$ & 0.064 \\
\hline Age (per year increase) & 1.01 & $0.97-1.05$ & 0.727 \\
\hline Female sex & 0.30 & $0.095-0.97$ & $0.044^{*}$ \\
\hline Admission NIHSS (per point increase) & 0.97 & $0.88-1.06$ & 0.474 \\
\hline Admission ASPECTS (per point increase) & 0.78 & $0.58-1.06$ & 0.110 \\
\hline Symptom-onset/last-seen well to reperfusion (per minute increase) & 1.00 & $0.99-1.00$ & 0.580 \\
\hline $\mathrm{TICl}$ (per grade increase) & 0.43 & $0.20-0.94$ & $0.035^{*}$ \\
\hline History of diabetes & 1.16 & $0.41-3.24$ & 0.783 \\
\hline History of coronary artery disease & 1.25 & $0.44-3.53$ & 0.672 \\
\hline \multicolumn{4}{|l|}{ Anticoagulants } \\
\hline No anticoagulants & Baseline & Baseline & Baseline \\
\hline Vitamin K antagonists & 1.05 & $0.11-10.13$ & 0.963 \\
\hline Direct oral anticoagulants & 3.08 & $0.55-17.28$ & 0.201 \\
\hline \multicolumn{4}{|l|}{ Antiplatelet medication } \\
\hline No antiplatelet medication & Baseline & Baseline & Baseline \\
\hline Aspirin & 2.30 & $0.74-7.09$ & 0.146 \\
\hline Aspirin+other antithrombotic & Did not converge & Did not converge & Did not converge \\
\hline Admission imaging modality CT vs. MRI & 0.52 & $0.14-1.92$ & 0.328 \\
\hline IV tPA & 2.33 & $0.75-7.17$ & 0.142 \\
\hline \multicolumn{4}{|l|}{ Site of intracranial occlusion } \\
\hline Intracranial ICA & Baseline & Baseline & Baseline \\
\hline M1 & 0.27 & $0.08-0.86$ & $0.026^{*}$ \\
\hline M2 & 0.29 & $0.07-1.14$ & 0.077 \\
\hline
\end{tabular}

$\mathrm{M} 1 / \mathrm{M} 2$, first and second segment of the middle cerebral artery, respectively.

aOR, adjusted odds ratio; Cl, confidence interval; IA, intra-arterial; UK, Urokinase; tPA, tissue plasminogen activator; NIHSS, National Institutes of Health Stroke Scale; ASPECTS, Alberta Stroke Program Early CT Score; TICl, thrombolysis in cerebral infarction; CT, computed tomography; MRI, magnetic resonance imaging; IV, intravenous; ICA, internal carotid artery.

${ }^{*} P<0.05$.

Supplementary Table 2. Risk of symptomatic intracranial hemorrhage with strata of prior antithrombotic and oral anticoagulant intake

\begin{tabular}{lcc}
\hline & No slCH & sICH \\
\hline Antiplatelet medication* & & $18(8.3)$ \\
No antiplatelet medication & $199(91.7)$ & $9(11.4)$ \\
Aspirin & $70(88.6)$ & $0(0)$ \\
Aspirin+another antiplatelet medication (e.g., prasugrel) & $9(100)$ & 0.59 \\
$P$ & & $21(8.2)$ \\
Oral anticoagulants ${ }^{\dagger}$ & $126(91.8)$ & $2(8.3)$ \\
No oral anticoagulant medication & $22(91.7)$ & $4(16.0)$ \\
Vitamin K antagonists & $21(84.0)$ & 0.44 \\
Direct oral anticoagulants & & \\
$P$ & &
\end{tabular}

Values are presented as number (\%). P-value calculated using Fisher's exact test.

sICH, symptomatic intracranial hemorrhage.

${ }^{*}$ Data available for 308 patients; ${ }^{+}$Data available for 309 patients. 
Supplementary Table 3. Any angiographic reperfusion improvement with strata of initial reperfusion success and occlusion site

\begin{tabular}{|c|c|c|c|}
\hline \multirow{2}{*}{ Strata } & \multicolumn{2}{|c|}{ Any angiographic reperfusion improvement } & \multirow{2}{*}{$P$} \\
\hline & - & + & \\
\hline \multicolumn{4}{|c|}{ TICl before IA fibrinolytics } \\
\hline 0 & $8(44.4)$ & $10(55.6)$ & 0.21 \\
\hline 1 & $4(40.0)$ & $6(60)$ & \\
\hline $2 a$ & $18(37.5)$ & $30(62.5)$ & \\
\hline $2 b$ & $82(53.9)$ & $70(46.1)$ & \\
\hline \multicolumn{4}{|l|}{ Site of occlusion } \\
\hline Intracranial ICA & $19(46.3)$ & $22(53.7)$ & 0.90 \\
\hline M1 & $64(50.4)$ & $63(49.6)$ & \\
\hline M2 & $29(48.3)$ & $31(51.7)$ & \\
\hline Total & 112 (49.6) & $116(50.9)$ & \\
\hline
\end{tabular}

Values are presented as number (\%). M1/M2, first/second segment of the middle cerebral artery. P-value were calculated using Fisher's exact test.

$\mathrm{TICl}$, thrombolysis in cerebral infarction; IA, intra-arterial; ICA, internal carotid artery.

Supplementary Table 4. Logistic regression model (dependent variable $\mathrm{mRS} \leq 2$ )

\begin{tabular}{|c|c|c|c|}
\hline Variable & $\mathrm{aOR}$ & $95 \% \mathrm{Cl}$ & $P$ \\
\hline IA UK vs. IA tPA & 1.35 & $0.87-2.98$ & 0.178 \\
\hline Age (per year increase) & 0.94 & $0.91-0.96$ & $<0.001^{*}$ \\
\hline Female sex & 0.58 & $0.28-1.21$ & 0.148 \\
\hline Admission NIHSS (per point increase) & 0.88 & $0.83-0.94$ & $<0.001^{*}$ \\
\hline Admission ASPECTS (per point increase) & 1.05 & $0.84-1.31$ & 0.686 \\
\hline Symptom-onset/last-seen well to reperfusion (per minute increase) & 0.99 & $0.99-1.00$ & 0.087 \\
\hline $\mathrm{TICl}$ (per grade increase) & 2.36 & $1.26-4.13$ & $0.007^{*}$ \\
\hline History of diabetes & 0.38 & $0.16-0.91$ & $0.030^{+}$ \\
\hline History of coronary artery disease & 0.22 & $0.10-0.49$ & $<0.001^{*}$ \\
\hline \multicolumn{4}{|l|}{ Anticoagulants } \\
\hline No anticoagulants & Baseline & Baseline & Baseline \\
\hline Vitamin $\mathrm{K}$ antagonists & 0.88 & $0.22-3.46$ & 0.855 \\
\hline Direct oral anticoagulants & 0.09 & $0.01-0.56$ & $0.009^{*}$ \\
\hline \multicolumn{4}{|l|}{ Antiplatelet medication } \\
\hline No antiplatelet medication & Baseline & Baseline & Baseline \\
\hline Aspirin & 1.05 & $0.45-2.43$ & 0.918 \\
\hline Aspirin+other antithrombotic & 1.28 & $0.21-7.67$ & 0.791 \\
\hline Admission imaging modality CT vs. MRI & 2.38 & $0.99-5.68$ & 0.051 \\
\hline IV tPA & 1.35 & $0.63-2.87$ & 0.442 \\
\hline \multicolumn{4}{|l|}{ Site of intracranial occlusion } \\
\hline Intracranial ICA & Baseline & Baseline & Baseline \\
\hline M1 & 1.99 & $0.73-5.44$ & 0.175 \\
\hline M2 & 5.22 & $1.67-16.27$ & $0.004^{*}$ \\
\hline
\end{tabular}

$\mathrm{M} 1 / \mathrm{M} 2$, first and second segment of the middle cerebral artery, respectively.

mRS, modified Rankin Scale; aOR, adjusted odds ratio; Cl, confidence interval; IA, intra-arterial; UK, urokinase; tPA, tissue plasminogen activator; NIHSS, National Institutes of Health Stroke Scale; ASPECTS, Alberta Stroke Program Early CT Score; TICl, thrombolysis in cerberal infarction; IV, intravenous; ICA, internal carotid artery.

${ }^{*} P<0.01 ;{ }^{+} P<0.05$. 